NeuroImage, 34, 4, 2007, 1692-1707

\title{
Top-down processes during auditory phoneme categorization in dyslexia: A PET study
}

\author{
O. Dufor, ${ }^{\mathrm{a}, *}$ W. Serniclaes, ${ }^{\mathrm{b}}$ L. Sprenger-Charolles, ${ }^{\mathrm{b}}$ and J.-F. Démonet ${ }^{\mathrm{a}}$ \\ a INSERM Unit 455, Université Paul-Sabatier and Department of Neurology, CHU Purpan, 31059 Toulouse, France \\ ${ }^{\mathrm{b}}$ CNRS UMR 8606 and Université René Descartes, France \\ Received 10 January 2006; revised 18 September 2006; accepted 30 October 2006
}

While persistence of subtle phonological deficits in dyslexic adults is well documented, deficit of categorical perception of phonemes has received little attention so far. We studied learning of phoneme categorization during an activation $\mathrm{H}_{2} \mathrm{O}^{15}$ PET experiment in 14 dyslexic adults and 16 normal readers with similar age, handedness and performance IQ. Dyslexic subjects exhibited typical, marked impairments in reading and phoneme awareness tasks. During the PET experiment, subjects performed a discrimination task involving sine wave analogues of speech first presented as pairs of electronic sounds and, after debriefing, as syllables /ba/ and /da/. Discrimination performance and brain activation were compared between the acoustic mode and the speech mode of the task which involved physically identical stimuli; signal changes in the speech mode relative to the acoustic mode revealed the neural counterparts of phonological topdown processes that are engaged after debriefing. Although dyslexic subjects showed good abilities to learn discriminating speech sounds, their performance remained lower than those of normal readers on the discrimination task over the whole experiment. Activation observed in the speech mode in normal readers showed a strongly left-lateralized pattern involving the superior temporal, inferior parietal and inferior lateral frontal cortex. Frontal and parietal subparts of these left-sided regions were significantly more activated in the control group than in the dyslexic group. Activations in the right frontal cortex were larger in the dyslexic group than in the control group for both speech and acoustic modes relative to rest. Dyslexic subjects showed an unexpected large deactivation in the medial occipital cortex for the acoustic mode that may reflect increased effortful attention to auditory stimuli. (C) 2006 Elsevier Inc. All rights reserved.

\section{Introduction}

According to preponderant theories, developmental dyslexia, a specific deficit of leaming to read and spell, relates to impaired phonological representations to which efficient mapping of homologous written components cannot be stabilized and automatized (Frith, 1999). At variance with other disorders of language

\footnotetext{
* Corresponding author. Fax: +5 61499524 .

E-mail address: Olivier.Dufor@toulouse.inserm.fr (O. Dufor).

Available online on ScienceDirect (www.sciencedirect.com).
}

processing described in dyslexia, impairment of categorical perception of phonemes (Serniclaes et al., 2001) and particularly the abnormalities of its neural counterparts have received only little attention so far (Ruff et al., 2002, 2003) (for a review, see Demonet et al., 2004). Disorders of categorical perception of phonemes imply defective discrimination between phonetically close phoneme pairs and, possibly, abnormal neural substrates of the corresponding phonological representations. In dyslexic subjects, it may be that the neural coding of each phonological representation share too many components with the other or that the corresponding neural counterparts cannot be activated differentially (Labatut et al., 2004).

Though categorical perception per se is a widely shared ability among mammal species, phoneme categorization is crucial to speech perception as it allows subjects to group under the same phonological representation an open set of acoustically variable stimuli, as rich as the number of people who could pronounce them (Liberman et al., 1957). For normal readers, well-categorized phonological representations stored in perceptual long-term memory constitute the basic repertoire with which written material can be associated.

Categorical perception is a dynamic phenomenon that shows leaming effects so that subjects exposed to unfamiliar stimuli become progressively able to associate them with known speech sounds (for details, see Semiclaes et al., 2001; Sprenger-Charolles et al., 2006). The study of such learning-related effects on both behavioral performance and neuro-imaging signals constitutes an experimental framework that is suitable for appraising the topdown influence of long-acquired phonological representations on the categorization of unfamiliar, ambiguous acoustic stimuli. Experiments of this type have been conducted by Serniclaes and colleagues (2004) in normal and dyslexic subjects who were presented with ambiguous synthetic stimuli prepared with sine waves mimicking $/ \mathrm{ba} /$ and $/ \mathrm{da} /$ syllables and asked to discriminate them in stimulus pairs. Although immediately perceived as electronic sounds resembling short whistles ("non-speech mode" or "acoustic"), these sounds were in most cases recognized as syllables after short training and debriefing ("speech mode") as a result of phonological top-down processes that facilitate stimulus 
categorization. Dehaene-Lambertz et al. (2005) explored the neural counterparts of this phonological training using fMRI in normal subjects. This experiment involved physical variations of stimuli that yielded either phonemic boundary crossing (e.g., from /ba/ to / $\mathrm{da} /$ ) or acoustic variants of the same phoneme (e.g., from /ba975/ to /ba1250/ depending on the frequency of a specific sine wave), respectively, "between-category" and "within-category" pairs. Topdown effects were revealed by the "speech mode" against "acoustic mode" comparison while categorical perception effect relied on the "between-category against within-category" comparison. The neural counterparts of these effects consisted in a massive engagement of several left-sided peri-sylvian regions when subjects have learned to categorize stimuli as distinct phonemes.

In dyslexic subjects, abnormalities of phonological representations should have detectable effects on behavioral and neuroimaging changes measured over a neuro-imaging experiment involving a phonological learning similar to that used by Dehaene-Lambertz et al. (2005) in their fMRI study. Here we used PET to investigate the neural correlates of phonological learning in dyslexic subjects as well as control normal readers since this neuroimaging technique offers a quieter acoustic environment and was more suitable for exploring the substrates of a speech discrimination task that subjects with language impairments might find challenging. The PET method implied using a blocked design involving different stimulus pairs in the same blocks, at variance with the event-related design used by Dehaene-Lambertz et al. (2005). While we could not directly compare the neural correlates of perception of the different stimulus pairs (e.g., between- and within-category), the present study focused on the neural correlates of phonological top-down processes, i.e., the comparison of brain activation patterns observed in the dyslexic and the control groups during perception of physically identical stimuli, processed respectively in the acoustic and speech modes of the experiment. Based on previous imaging studies of speech perception in dyslexic subjects, we hypothesized that in the speech mode fewer activation will be found relative to control readers in the left superior temporal / inferior parietal cortex, especially the supra-marginal gyrus (Simos et al., 2002; Ruff et al., 2002, 2003, Eden et al., 2004); in normal subjects, these regions were found to be more active for the speech mode than the acoustic mode in the study by Dehaene-Lambertz et al. (2005). Furthermore, some previous studies on speech processing in dyslexia suggested that some right hemispheric regions, especially in the temporal cortex might be more involved than the homologous left-sided regions (see for instance McCrory et al., 2000; Simos et al., 2000, 2002; Giraud et al., 2005), reflecting an atypical preponderance of language processes in dyslexic subjects or adaptive mechanisms that developed as a consequence of defective functions of the left hemispheric territories.

\section{Methods}

\section{Subjects}

Fourteen adult subjects (mean age $30 \pm 9.8$ years) showing typical sequelae of developmental phonological dyslexia and sixteen normal readers (mean age $27.6 \pm 5.1$ years) were involved. All subjects were physically healthy and free of neurological disease, head injury, psychiatric disorders and hearing deficit on tonal audiometry. They were all men, French native speakers, had normal performance IQ (score $>90$ on Wechsler Adult Intelligence
Scale, WAIS-III) and achieved high school level. Four dyslexic subjects and three control subjects were left-handed according to the Edinburgh Inventory.

\section{Inclusion/exclusion criteria}

Diagnosis of dyslexia. Dyslexic subjects had a documented history of reading difficulties during childhood and received speech therapy over variable periods. They were diagnosed in accordance with the guidelines of the WHO ICD-10.

The following tests were used to assess language and working memory: reading of regular, irregular and "loan" words (e.g., "yacht" in French), rapid digit naming, phoneme awareness tasks (notably phoneme deletion, rhyme judgment and spoonerisms) and auditory word span for 1-syllable and 4-syllable words.

From a previously established procedure, four behavioral tests were used to further ascertained the diagnosis of developmental dyslexia: reading regular words (cut-off latency between stimulus appearance on a computer screen and onset of subject's oral response $660 \mathrm{~ms}$ ), reading legal pseudo-words (cut-off latency $940 \mathrm{~ms}$ ), reading aloud 50 digits (mean time cut-off $18 \mathrm{~s}$ ), spelling on dictation of 15 irregular frequent words (cut-off $>3$ errors). Cut-off score was defined following a preexperimental study involving a separate group of 18 adult dyslexics and 65 controls; these criteria are those used in the European Dyslexia Study, as described by Paulesu et al. (2001) and were also used by Ruff et al. $(2002,2003)$ and by Silani et al. (2005). Subjects were considered dyslexic when they scored out of cut-off in at least two of the four tests.

Subjects involved in the control group did not match any of the items of the systematic inventory of dyslexia, had no history of learning disabilities in childhood and scored in the normal range on the above listed tests used to diagnose dyslexia

In addition, the " $\mathrm{d} 2$ " test, a visual test for sustained attention (Brickenkamp, 1994) was used to exclude subjects presenting a developmental disorder of attention; only subjects scoring on the $\mathrm{GZ}$ index in the upper two quartiles were included.

PET scanning. Subjects were scanned in a darkened room with eyes closed. Their head was immobilized and head position was aligned transaxially to the orbitomeatal line with a laser beam and controlled before each acquisition. Measurements of regional distribution of radioactivity were performed with an ECAT HR+ (Siemens ${ }^{8}$ ) PET camera with full volume acquisition (63 planes, thickness $2.4 \mathrm{~mm}$, axial field-of-view $158 \mathrm{~mm}$, in-plane resolution $\approx 4.2 \mathrm{~mm}$ ). The duration of each scan was $80 \mathrm{~s}$; about $6 \mathrm{mCi}$ of $\mathrm{H}_{2} \mathrm{O}^{15}$ was administered to each subject. Stimulation on the experimental conditions was started $\approx 20 \mathrm{~s}$ before data acquisition and continued until scan completion.

MRI scanning. All subjects were scanned at the Neuroradiology Department of Toulouse Purpan hospital on a Siemens Magnetom Vision (1.5 Tesla). After sagittal localization images, 256 contiguous T1-weighted images were obtained parallel to the intercommissural plane covering the all head $(256 \times 256$ matrix, $1.17 \mathrm{~mm}^{3}$ voxels)

Data recording. Digitized stimuli and response recording were run on a PC using the Presentation ${ }^{\circledR}$ software to deliver stimuli and monitor response accuracy and reaction time.

PET images obtained were processed with Matlab 6.5 (MatWorks, Natick, MA, USA) and the Statistical Parametric Mapping 
software (SPM2, Wellcome Department of Imaging Neuroscience, London, UK, 2002, www.fil.ion.ucl.ac.uk).

\section{Stimuli and paradigm}

Dyslexic subjects and normal readers were asked to produce same/different responses by clicking on either the right or the left button of a PC mouse to pairs of auditory stimuli made of sine wave speech (/ba/ and /da/ syllables) (see Serniclaes et al., 2001; DehaeneLambertz et al., 2005, for details). The 'same' responses were associated with either the right or the left mouse button in balanced subgroups of the two groups of control or dyslexic subjects.

We used stimuli made from sine wave analogues of a (consonant+vowel/a/) syllables wherein the consonant was varied along a place-of-articulation continuum. The endpoints were given appropriate values for perception of a / ba/ syllable at one end and for the perception of $\mathrm{a} / \mathrm{da} /$ syllable at the other end. The difference in place of articulation between two initial consonants was created by modifying the onset of the initial frequency transition (SIN2 and SIN3), which corresponded to those of the second and the third formant in natural speech (F2 and F3). This onset frequency varied from $975 \mathrm{~Hz}$ to $1800 \mathrm{~Hz}$ in three equal steps of $275 \mathrm{~Hz}$ for SIN2 and from $1975 \mathrm{~Hz}$ to $3400 \mathrm{~Hz}$ in three equal steps of $475 \mathrm{~Hz}$ for SIN3. The end frequencies of SIN2 and SIN 3 transition were fixed at $1300 \mathrm{~Hz}$ and $2500 \mathrm{~Hz}$, respectively. The stimuli were labeled according to their phonemic identity (i.e., depending on whether they were predominantly identified as $/ \mathrm{ba} / \mathrm{or} / \mathrm{da} /$ ) and to the onset of SIN2: ba975, ba1250, da1525 and da1800. The initial frequency of the lowest formant (F1) was $100 \mathrm{~Hz}$, and its end frequency was $750 \mathrm{~Hz}$. The VOT was $60 \mathrm{~ms}$, the duration of all frequency transition was $40 \mathrm{~ms}$ and the duration of the stable vocalic segment was $170 \mathrm{~ms}$ including this period of transition.

Stimuli were presented in pairs with an ISI of $100 \mathrm{~ms}$ and an available time for response of $1440 \mathrm{~ms}$, yielding an SOA of $2 \mathrm{~s}$.

Two different versions of this continuum were constructed, differing only according to the synthesis method, either pure sine wave synthesis (SWac and SWsp) or pitch-modulated sine wave synthesis. The latter was obtained by adding low frequency amplitude modulation to the sine wave sounds and called SWsp+F0. F0 modulation made the stimuli appearing clearly as synthetic speech samples (see Semiclaes et al., 2001, for details).

Pairs were made of either the same stimulus $\left(\mathrm{BA}_{1} / \mathrm{BA}_{1} ; \mathrm{DA}_{1} /\right.$ $\mathrm{DA}_{1} ; \mathrm{BA}_{2} / \mathrm{BA}_{2}$ and $\mathrm{DA}_{2} / \mathrm{DA}_{2}$ ), "within-category" stimuli (i.e., pairs in which stimuli differed in acoustic terms but pertained to the same phonological category, $\mathrm{BA}_{1} / \mathrm{BA}_{2} ; \mathrm{BA}_{2} / \mathrm{BA}_{1} ; \mathrm{DA}_{1} / \mathrm{DA}_{2}$ and $\mathrm{DA}_{2} / \mathrm{DA}_{1}$ ) or "between-category" stimuli (i.e., pairs in which stimuli differed in phonological terms, $\mathrm{BA}_{1} / \mathrm{DA}_{2}$ and $\mathrm{DA}_{2} / \mathrm{BA}_{1}$ ).

The PET procedure involved 12 runs including 3 runs (or sessions) for 3 experimental tasks, SWac, SWsp and SWsp with F0, interspersed with 3 rest runs without auditory stimulation.

Each experimental run was made of 72 pairs of stimuli pseudorandomly distributed among the three types of pairs, with 24 SAME $\left(6 \mathrm{BA}_{1} / \mathrm{BA}_{1} ; 6 \mathrm{DA}_{1} / \mathrm{DA}_{1} ; 6 \mathrm{BA}_{2} / \mathrm{BA}_{2}\right.$ and $\left.6 \mathrm{DA}_{2} / \mathrm{DA}_{2}\right), 24$ WITHIN $\left(6 \mathrm{BA}_{1} / \mathrm{BA}_{2} ; 6 \mathrm{BA}_{2} / \mathrm{BA}_{1} ; 6 \mathrm{DA}_{1} / \mathrm{DA}_{2}\right.$ and $\left.6 \mathrm{DA}_{2} / \mathrm{DA}_{1}\right)$ and 24 BETWEEN (12BA $/ \mathrm{DA}_{2}$ and $\left.12 \mathrm{DA}_{2} / \mathrm{BA}_{1}\right)$ pairs.

Subjects were asked to listen carefully and detect possible differences between stimuli within pairs.

In the first 3 task runs (SWacoustic or "SWac" condition), subjects were told that they will perform an auditory task involving electronic sounds, avoiding any reference to language processing.
After these first 3 runs, subjects were debriefed and instructed that the stimuli were in fact synthetic syllables. They were trained to discriminate well between these synthetic $/ \mathrm{ba} /$ versus $/ \mathrm{da} /$ syllables. For the sake of rapid training efficacy, subjects were trained with either SAME or BETWEEN pairs, presented in six short sessions of twenty pairs, alternating sessions with and without $\mathrm{F} 0$.

All subjects quickly reached a $75 \%$ of accurate judgment using these stimuli.

Then, in addition to rest runs, the PET experiment further involved the other two experimental conditions, each involving three runs, SWsp and SWsp+F0. Subjects were equally distributed in two subgroups according to whether the SWsp condition was administered before ( 8 control subjects, 7 dyslexic subjects) or after ( 8 control subjects, 7 dyslexic subjects) the SWsp + F0 condition . Before each experimental condition, a demonstration of twenty pairs of sounds (10 SAME and 10 BETWEEN) were presented involving stimuli with or without F0 to keep subjects familiar with the task.

The study was approved by the local Ethics committee and all subjects gave written informed consent.

\section{Data analysis}

Analysis of behavioral responses involved accuracy scores consisting of $\%$ of "same" responses to SAME pairs, $\%$ of "different" responses to BETWEEN pairs and \% of "different" responses for WITHIN pairs. It should be noted that for the latter pair type the assignment of accuracy to 'different' responses is rather arbitrary as it refers to differences in acoustic terms whereas, in consideration of categorical perception, one would expect 'same' responses to be the correct answers.

Neuroimaging data were analyzed with SPM2. Images were realigned using the first scan as reference and then transformed into the standard space of the Montreal Neurological Institute template and smoothed with a 8-mm Gaussian filter. First, across-condition contrasts were obtained in each individual (threshold $P<0.001$ ). Then random-effect analyses were conducted for across-condition contrasts in each group (e.g., (SWac-rest)) with an uncorrected threshold set at $P<0.001$ and a cluster extent $k>30$ voxels. Betweengroups comparisons (e.g., [SWsp-rest $]_{\text {cont }}$ vs. [SWsp-rest $]_{\text {dys }}$ ) used a threshold at $P<0.05$ and a cluster extent $k>30$.

In general, between-groups compound contrasts were analyzed using an inclusive mask (threshold $P=0.05$ ) which consisted of the second term of the contrast to isolate increase of activation in the first term over and above activations in the masking contrast. Therefore, any significant cluster in the compound contrast reflect significant activation in both groups and a surplus of activity in the group considered in the first term.

As the neural correlates of phonological top-down processing have never been examined in developmental dyslexia following leaming, the exploration of the present neuroimaging data was systematic and involved two main sections, analyses of effects in each group of subjects and analyses between groups.

For both within-group and between-group analyses, we considered in tum effects of each task compared to rest and comparisons between tasks (e.g., speech vs. acoustic). Contrasts comparing either acoustic or speech perception against rest are important to address deactivation effects and brain activity changes related to attention modulation. As mentioned in the introduction, the direct comparison between the acoustic (SWac) and the speech 
Table 1

Mean score comparison between the dyslexic group and the control group for the four neuropsychological tests forming inclusion criteria

\begin{tabular}{|c|c|c|c|}
\hline $\begin{array}{l}\text { Number of errors }{ }^{1} \text { or } \\
\text { reaction time } \mathrm{e}^{2}(\mathrm{~ms})\end{array}$ & Dyslexics & Controls & $\begin{array}{l}P \text { value } \\
\text { (Mann-Whitney } U \text { test }\end{array}$ \\
\hline IWS $^{1}$ & $6.23 \pm 3.19$ & $3.25 \pm 2.67$ & $0.013^{*}$ \\
\hline $\mathrm{NWR}^{2}$ & $1060.12 \pm 320.66$ & $619.65 \pm 92.251$ & $0.000^{*}$ \\
\hline $\mathrm{DN}^{2}$ & $21.13 \pm 3.15$ & $14.88 \pm 1.97$ & $0.000^{*}$ \\
\hline
\end{tabular}

IWS: irregular word spelling, WR: word reading, NWR: non-word reading, DN digit naming.

(SWsp) modes of the experiment allowed us to explore the brain correlates of top-down phonological processes while heard stimuli were physically identical. These effects were formally tested as the interaction between group effect (control/dyslexic subjects) and mode effect (speech/acoustic). This interaction was assessed as the outcome of the compound contrast ([SWsp-SWac $]_{\text {cont }}$ vs. [SWspSWac $\left.]_{\text {dys }}\right)$. At variance with other compound contrasts analyzed in this study (see above), this contrast was masked inclusively $(P=0.05)$ with the first term as decreased activation was expected in the dyslexic group on the basis of previous findings of reduced brain activities for speech perception in developmental dyslexia (for a review, see Temple, 2002).

\section{Results}

\section{Performance on neuropsychological tests}

As generally reported (Paulesu et al., 2001), dyslexics performed significantly worse than controls in reading, short-memory and phonological tasks, in spite of normal IQ and attention capacities. In reading tests as well as in phoneme awareness tests we observed a significant difference between the two groups not only in terms of response accuracy but also in terms of reaction time.

Table 1 summarizes the results on tests used as inclusion criteria. Strong differences were observed between the two groups for reaction times while a less marked but still significant difference existed for spelling irregular words on dictation.

Table 2 shows performance on other language tests that demonstrated significant deficits in dyslexic subjects relative to control subjects except for long-word span and non-word spelling tasks. Performance IQ did not differ in the two groups.

Performance on the speech sound task during the PET session

We performed two ANOVAs with repeated measures on accuracy scores and reaction time considering the two groups across twenty seven sessions subdivided in three within-subject factors ( 3 pair types, 3 Conditions and 3 sessions), group (normal vs. dyslexic) being considered an independent variable.

For accuracy scores, the ANOVA with repeated measures showed a significant group main effect $[F(1,25)=4,36, P=0.047]$ as control subjects showed slightly better performance than dyslexic subjects.

Strong main effects for each within-subject factor were obtained: Pairs: $[F(2,24)=244,359, P<0.001]$, Condition $[F(2,24)=155,282$, $P<0.001]$ and Session $[F(2,24)=5,791, P=0.005]$. Interaction effects were also observed: Pairs by Condition $[F(4,22)=56,872$, $P<0.001]$, Pairs by Session $[F(4,22)=3,76, P=0.009]$. These effects mainly related to differences between naive and postdebriefing conditions. A (SWac vs. SWsp) $\times$ Pair types (BETWEEN vs. WITHIN) interaction contrast was observed showing a higher increase of "different" responses to the BETWEEN pair types than to the WITHIN pairs in SWsp relative to SWac $[F(1,28)=46,77$, $P<0.000]$. Dramatic improvement of performance was observed after debriefing for the BETWEEN pairs (Fig. 1) in both groups. Before debriefing, stimuli pairs were not discriminated and even the BETWEEN pairs gave rise to 'same' responses in most cases; the condition effect on the BETWEEN pairs was highly significant $[F$ $(1,28)=143,01, P<0.001]$. Table 3 presents the distribution of accuracy scores for each type of pairs over the experiment (for the sake of clarity, results were presented as if all subjects underwent $\mathrm{SWsp}+\mathrm{F} 0$ then SWsp). In most cases, control subjects performed better than dyslexic subjects for non-"different" pairs (BETWEEN and SAME pairs). For WITHIN pairs, the number of responses 'different' increased after debriefing; planned comparisons showed highly significant differences in the dyslexic group $[F(1,25)=13.28$ $P=0.001]$ while difference was less marked in the control group $[F$ $(1,25)=6.06 P=0.021]$.

For reaction times, a repeated measures ANOVA showed significant main effects of Pairs $[F(2,56)=28,12, P<0.001]$ and Condition $[F(2,56)=31,828, P<0.001]$ and interactions: Pair types

Table 2

Mean score comparison between the dyslexic group and the control group for the overall neuropsychological tests battery

\begin{tabular}{|c|c|c|c|}
\hline \multirow{2}{*}{$\begin{array}{l}\text { Score }^{1} \text { or } \\
\text { number of errors }\end{array}$} & \multirow{2}{*}{$\begin{array}{l}\text { Dyslexics } \\
\begin{array}{l}\text { Mean } \pm \text { standard } \\
\text { deviation }\end{array}\end{array}$} & \multirow{2}{*}{ 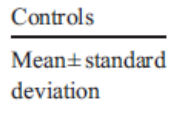 } & \multirow{2}{*}{$\begin{array}{l}P \text { value } \\
\text { (Mann- } \\
\text { Withney } \\
U \text { test) }\end{array}$} \\
\hline & & & \\
\hline QI $_{\text {global }}{ }^{1}$ & $105.06 \pm 12.52$ & $114.66 \pm 8.76$ & $0.020^{*}$ \\
\hline QI performance ${ }^{1}$ & $106.20 \pm 9.27$ & $108.66 \pm 10.10$ & 0.480 \\
\hline $\mathrm{QI}_{\text {verbal }}{ }^{1}$ & $104.06 \pm 14.00$ & $116.26 \pm 12.31$ & $0.015^{*}$ \\
\hline LWSpan & $3.01 \pm 1.70$ & $3.31 \pm 1.12$ & 0.538 \\
\hline SWSpan & $3.11 \pm 1.14$ & $5.04 \pm 0.52$ & $0.000^{*}$ \\
\hline SYL & $1.61 \pm 1.44$ & $0.56 \pm 0.89$ & $0.023^{*}$ \\
\hline PHO & $6.76 \pm 5.41$ & $1.43 \pm 2.68$ & $0.001^{*}$ \\
\hline Spo & $3.25 \pm 2.98$ & $0.75 \pm 1.39$ & $0.007^{*}$ \\
\hline PHO & $6.61 \pm 3.30$ & $3.43 \pm 2.06$ & $0.003^{*}$ \\
\hline NWS & $2.69 \pm 2.39$ & $1.56 \pm 1.09$ & 0.301 \\
\hline WR & $1.76 \pm 2.08$ & $0.18 \pm 0.40$ & $0.006^{*}$ \\
\hline NWR & $6.07 \pm 3.90$ & $1.56 \pm 1.31$ & $0.000^{*}$ \\
\hline LWR & $5.00 \pm 3.05$ & $0.81 \pm 1.04$ & $0.000^{*}$ \\
\hline IWR & $6.41 \pm 4.69$ & $0.25 \pm 0.68$ & $0.000^{*}$ \\
\hline Rhymes & $9.07 \pm 4.29$ & $5.06 \pm 2.90$ & $0.009^{*}$ \\
\hline
\end{tabular}

Mean, standard deviation and $P$ value (U test of Mann-Withney), for the analysis of performances on the neuropsychological tests in each group. LWSpan : long words span, SWSpan : short words span, -SYL : syllabic subtraction, -PHO : phonemic subtraction, Spo : Spoonerisms, PHO : phonemic categorisation, NWS : non-word spelling, WR : word reading, NWR : non-word reading, LWR: loan word reading, IWR : irregular word reading and Rhymes : rhymes judgment. 


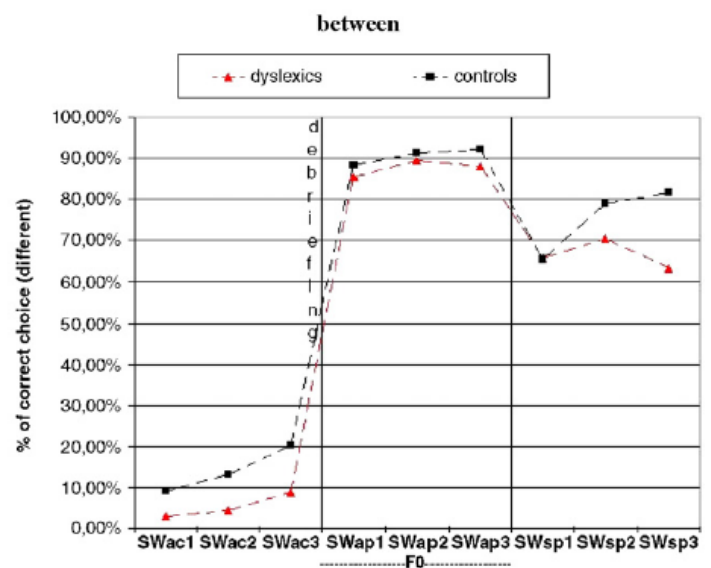

Fig. 1. Percentage of behavioral responses for the «between» type of stimul along the experiment.

by Condition $[F(4,112)=19,97, P<0.001]$ and Condition by Session $[F(4,112)=2,83, P=0.025]$. The former effects mainly related to RT increase in post-debriefing conditions (SWsp and $\mathrm{SWsp}+\mathrm{F} 0$ ) relative to the naive (SWac) condition. Concerning the Condition by Session interaction, the effect was mainly due to a RT increase over the three sessions of SWac. About the Pairs by Condition interaction, a significant RT increase was observed for SAME pairs in SWsp relative to SWac whereas no change existed for BETWEEN pairs $[F(1,28)=50,144, P<0.001]$; the same effect was observed when opposing WITHIN and BETWEEN pairs $[F$ $(1,28)=7,89, P=0.008]$ and, to a lesser extent, SAME and WITHIN pairs $[F(1,28)=6,80, P=0.014]$.

Although group effect failed to reach significant threshold, an LSD Fisher's test showed a significant increase of responses latencies along the first three runs (SWac) in the control group only. $[P(\mathrm{SWac} 1 * \mathrm{SWac} 2)=0.007, P(\mathrm{SWac} 1 * \mathrm{SWac} 3)<0.001$ and $P$ $(\mathrm{SWac} 2 * \mathrm{SWac} 3)=0.028]$.

PET results

Contrasts between experimental conditions in each group of subjects

Control group. The comparison of the SWac condition relative to rest showed activation clusters which predominated in the right hemisphere and involved mainly the posterior part of the superior temporal gyrus, spreading to the posterior/inferior frontal cortex and the inferior parietal cortex, a symmetrical pattern, though reduced in extension, being observed in the left hemisphere.

In the SWsp-rest contrast, a clear effect of debriefing and leaming was a right-to-left shift of activation preponderance in the control group (Fig. 2). In this group, activations in the left hemispheric perisylvian cortex were predominant after subjects were informed of the linguistic nature of stimuli, relative to the naive SWac condition. In more details, the comparison of SWsp condition relative to rest showed activation clusters which predominated in the left hemisphere and involved the superior temporal gyrus, the inferior parietal lobule, the lower sensory-motor cortex and the posterior inferior premotor cortex ('Broca's area'). In the right hemisphere activation concerned the homologous areas but to a lesser extent, as clusters were reduced in extent and amplitude; they were located in the superior temporal gyrus, the inferior and middle frontal gyri and the inferior parietal cortex.

For both SWac and SWsp against rest, activations were seen in the upper part of the cerebellum bilaterally.

For both these contrasts also, deactivations were observed (clusters in green on Fig. 2) with a pattern very similar to what has been described in previous neuroimaging studies especially on language functions (Binder et al., 1999; Gusnard et al., 2001).

Similar results were brought up by the SWsp+F0-rest contrasts. The similarity of effects of SWsp $+F 0$ relative to $S W s p$ was observed over the whole set of results so that these effects will no longer be considered in the paper.

Results of the SWsp-SWac contrast (Fig. 3) revealed the neural counterparts of the phonological perception mode effect following debriefing and discrimination training. They consisted of bilateral activation in motor/premotor areas, the parietal cortex and the anterior cingulate cortex. The preponderance of activation in the left hemisphere was less obvious than in the above-described contrasts against rest. However, it was confirmed by the extent of the activated clusters in homologous regions. In the left parietal region 830 voxels were activated in the left cluster against 545 voxels in the right one (Fig. 3; green arrow) with similar $Z$ value for their peak. This left-sided preponderance was also observed in the frontal region with a cluster extending over 934 voxels in the left BA6 and BA9 regions against 184 voxels in the right homologous one. The peak $Z$ score of these clusters were also similar (i.e., 4.63 for the right frontal cluster and 4.31 for the left one).

Dyslexic group. For the SWac-rest comparison (Fig. 2), the activation clusters predominated in the right hemisphere, implicating, as in the control group, the posterior part of the superior temporal gyrus and the posterior/inferior frontal cortex; however,

Table 3

Mean percentage of performances for each group of subjects in each run for each type of pairs

\begin{tabular}{llrrrllllll}
\hline & & Run1 Sw & \multicolumn{1}{c}{ Run2 Sw } & Run3 Sw & Run1 Swsp+f0 & Run2 Swsp+f0 & Run3 Swsp+f0 & Run1 Swsp & Run2 Swsp & Run3 Swsp \\
\hline Between & dys & $3.07 \%$ & $4.81 \%$ & $8.79 \%$ & $85.36 \%$ & $89.37 \%$ & $88.00 \%$ & $65.61 \%$ & $70.52 \%$ & $63.38 \%$ \\
& tem & $9.23 \%$ & $13.20 \%$ & $20.27 \%$ & $88.15 \%$ & $91.35 \%$ & $92.02 \%$ & $65.51 \%$ & $78.78 \%$ & $81.56 \%$ \\
Same & dys & $94.92 \%$ & $93.30 \%$ & $90.11 \%$ & $89.96 \%$ & $88.99 \%$ & $85.86 \%$ & $73.98 \%$ & $78.49 \%$ & $74.67 \%$ \\
& tem & $89.45 \%$ & $89.92 \%$ & $83.32 \%$ & $92.19 \%$ & $90.63 \%$ & $88.27 \%$ & $77.83 \%$ & $80.77 \%$ & $82.19 \%$ \\
\multirow{2}{*}{ Within } & dys & $5.90 \%$ & $9.34 \%$ & $8.18 \%$ & $16.50 \%$ & $20.23 \%$ & $18.83 \%$ & $34.81 \%$ & $29.51 \%$ & $35.66 \%$ \\
& tem & $11.84 \%$ & $15.25 \%$ & $20.22 \%$ & $19.43 \%$ & $21.26 \%$ & $22.22 \%$ & $32.67 \%$ & $32.14 \%$ & $28.69 \%$ \\
\hline
\end{tabular}

Mean scores in each run and each group of subjects (rate of 'different' responses to BETWEEN pairs and to WITHIN pairs, rate of 'same' responses to SAME pairs). Note the large improvement of performance after debriefing. 

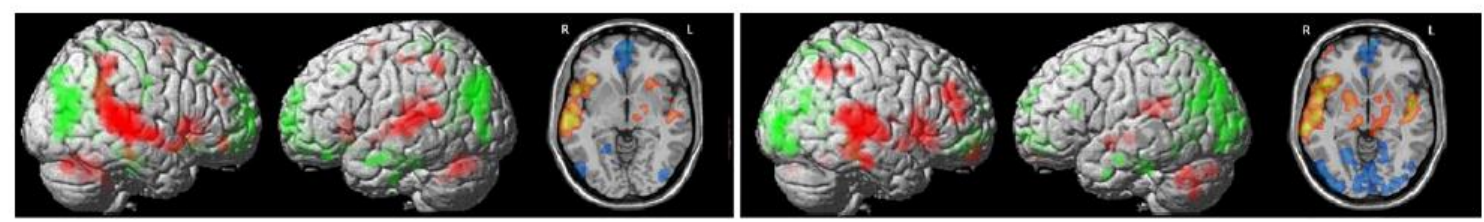

SWac- Rest Controls

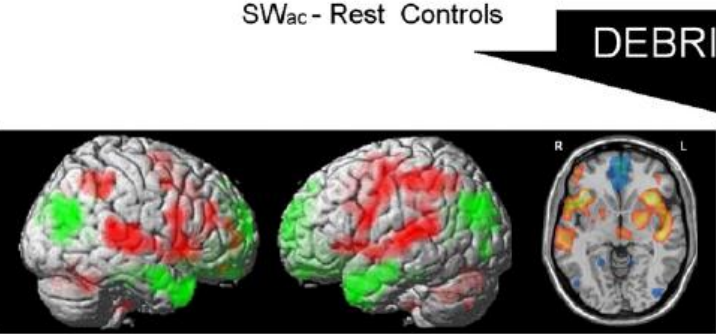

SWac-Rest Dyslexics
SWsp - Rest Controls

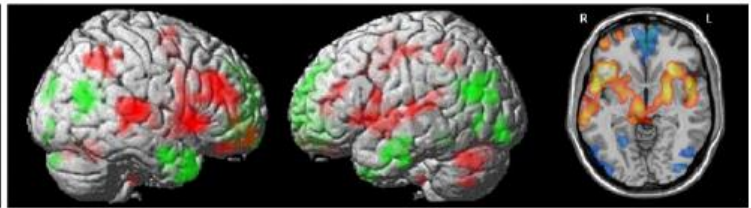

SWsp - Rest Dyslexics

Fig. 2. SWac minus rest contrast (top) and SWsp minus rest contrast (bottom) in each group of subjects. Control group on the left and dyslexic group shown on the right. Red (surface rendering) and "Hot" (slices) clusters represent activations while green (surface rendering) and "cold" (slices) clusters refer to deactivation. Coordinate for horizontal slice: $z_{\text {talairach }}=-8$. Abbreviations: $\mathrm{SWac}=$ sine wave syllables perceived as sounds before training; $\mathrm{SW} s \mathrm{sp}=\mathrm{same} \operatorname{sine}$ wave syllables as in SWac, perceived as speech after training (see text for details).

the pattem seemed to differ from that in controls as the cluster located in the inferior parietal cortex extended higher towards the upper part of the supra-marginal gyrus, and the right temporal cluster spread towards the middle temporal gyrus; finally, an additional cluster concerned the middle frontal gyrus.

In the SWsp-rest contrast (Fig. 2), an increase of activation in the left hemisphere was observed after debriefing with a pattern similar to that observed in the control group although the extent of activated clusters seemed diminished. An additional cluster was found in the left middle frontal gyrus. In the right hemisphere, the activation pattern is close to that observed in the control group.

For both contrasts, an activation in the upper part of the left cerebellar hemisphere was found.
As shown on Fig. 2, deactivations were observed in the dyslexic group; the pattern of these effects was roughly similar to that observed in the control group. However, even though subjects kept their eyes closed over the experiment, the SWac condition in dyslexic subjects elicited deactivation in the occipital cortex that was not observed in normal readers.

In the SWsp-SWac contrast (Fig. 3; Table 4), although the pattem seemed close to that observed in the control group, activated clusters appeared smaller. Activations were found in the left hemisphere in precentral, inferior frontal, claustrum/insula, postcentral, precuneus and posterior cingulate cortices, while they were located in the right hemisphere in precentral, anterior cingulate, cuneus and lingual/primary visual cortices.

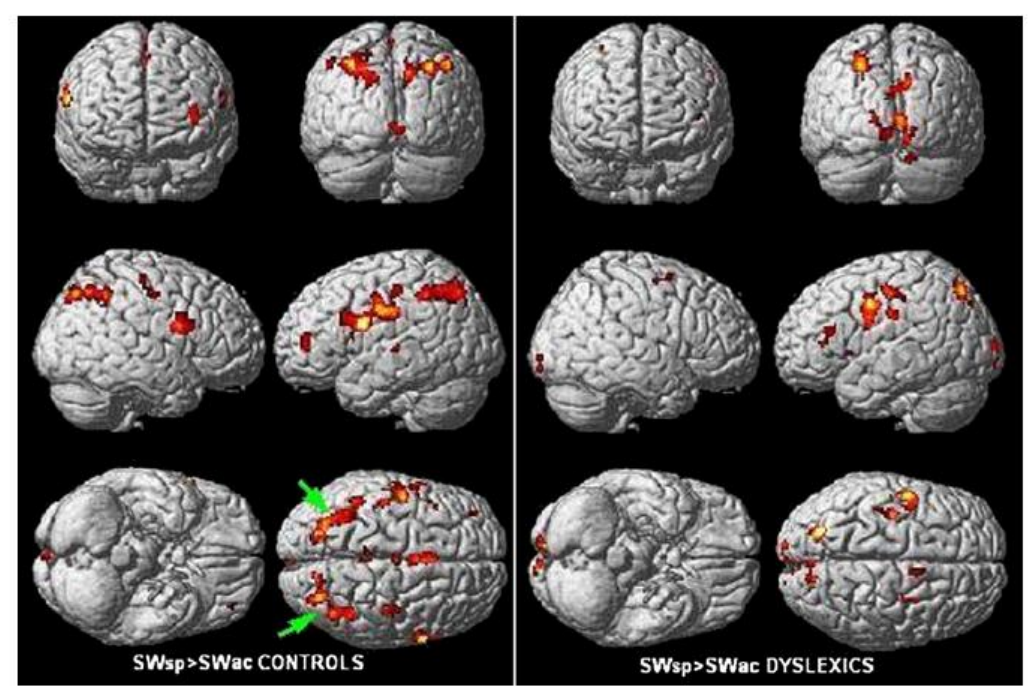

Fig. 3. SWsp minus SWac contrast in each group of subjects. Dyslexic group shown on the right and Control group on the left (abbreviations as in Fig. 2). 
Table 4

Higher activation in the SWsp condition than in the SWac condition for the dyslexics subjects (bottom) and for controls (top)

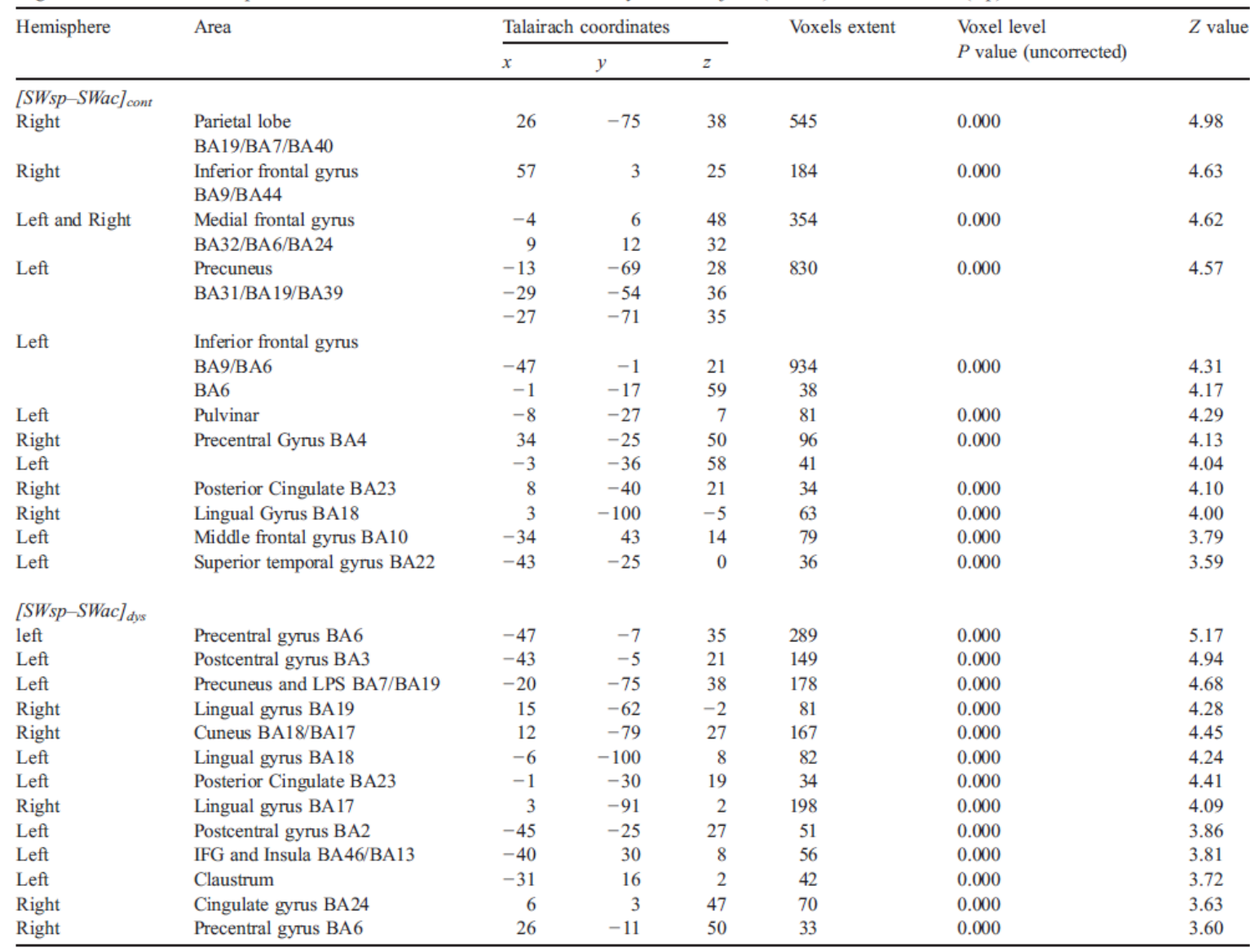

Only clusters corresponding to voxel level $P<0.001$ uncorrected were listed.

SWac $=$ acoustically perceived sine waves before training.

$\mathrm{SW} \mathrm{sp}=$ sine waves perceived as speech after training.

Therefore, activation in a large part of the medial occipital cortex (especially BA17) was found that was not noted in the control group.

These qualitative observations of the pattern of activation in the dyslexic group relative to the control group were further explored by a formal, statistical between-group analysis.

\section{Between-group contrasts}

Tasks against rest (Figs. 4A/B and 5A/B; Tables 5 and 6). The (Group $\times$ Condition) compound contrast (SWac-rest $]_{\text {cont }}$ vs [SWac-rest $]_{\text {dys }}$ ) (Fig. 4A; Table 5) showed that controls activated more than dyslexics the right superior temporal/supramarginal junction, as well as smaller parts of left-sided areas in the superior frontal and inferior parietal regions, and the superior part of the right cerebellar hemisphere.

In the reverse contrast ([SWac-rest $]_{\text {dys }}$ vs. [SWac-rest $]_{\text {cont }}$ ) (Fig. 4B; Table 5), dyslexics activated more than controls a large area in the right frontal cortex, a region of the right parietal cortex, as well as bilateral limbic structures, thalamus and putamen.
The (Group $\times$ Condition) compound contrast ([SWsp-rest ] cont vs. [SWsp-rest $]_{\text {dys }}$ ) (Fig. $5 \mathrm{~A}$; Table 6) showed that controls activated more than dyslexics a large area in the left hemisphere, encompassing frontal, temporal and parietal cortices, in the inferior and middle frontal gyri, the superior and middle temporal gyri and the superior and inferior parietal lobules.

In the reverse contrast ([SWsp-rest $]_{\text {dys }}$ vs. [SWsp-rest $\left.]_{\text {cont }}\right)$ (Fig. 5B; Table 6), activations were found in the right middle and inferior frontal areas homologous to Broca's area, in the right parahippocampal gyrus, as well as clusters in right thalamus, left putamen and both cerebellar hemispheres.

Comparison between tasks ( $S W s p$ vs. SWac). We first analyzed the results of the main effects of the interacting factors.

One factor consisted of the effect of perception mode whatever the groups, i.e., the opposition between the acoustic and the speech modes of the experiment allowing us to explore perception mode effects. This main effect just confirmed the large frontal-parietal pattern with left-sided predominance observed in each group separately (Fig. 6A) 
A

clusters activated for the contrast:
[ SWac- rest ]cont vs. [ SWac- rest]dys
B

clusters activated for the contrast:

[ SWac- rest ]dys vs. [ SWac- rest]cont
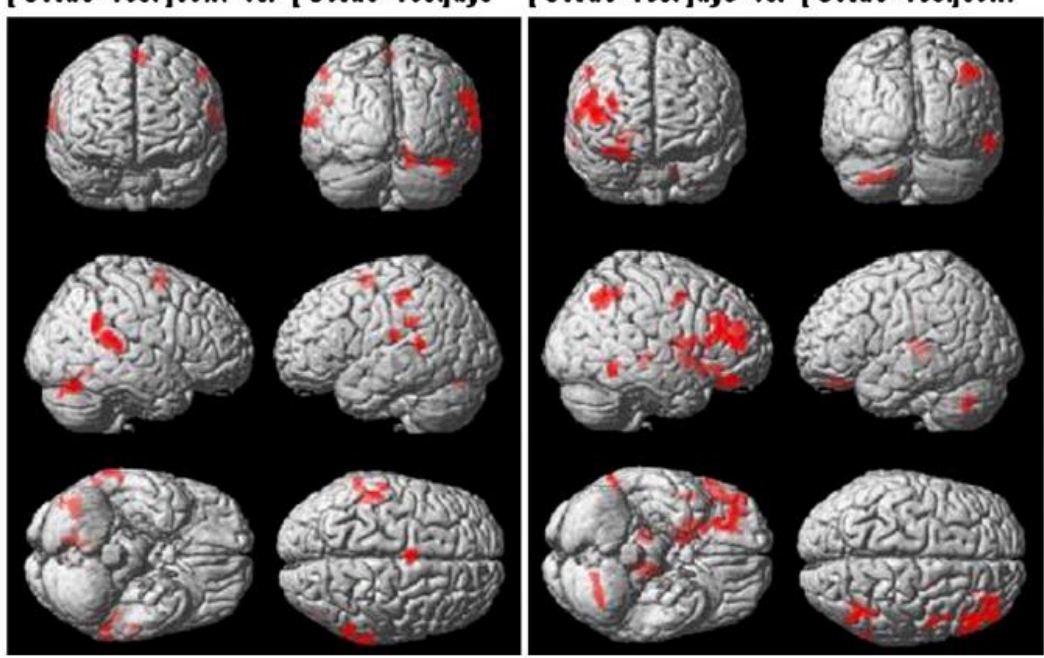

Fig. 4. Main clusters activated in the contrast image SWac minus Rest. Controls $>$ Dyslexics $(P=0.05)$ inclusively masked by SWac minus Rest in dyslexics (left) and Dyslexics $>$ Controls $(P=0.05)$ inclusively masked by SWac minus Rest in controls (right) (abbreviations as in Fig. 2).

The second factor was the group effect in both acoustic and speech modes of the experiment (Fig. 6B). The group main effect concerned few clusters; however, it revealed higher activity during both auditory tasks in control readers relative to dyslexic subjects in some small cortical regions, especially in the left inferior parietal lobule $(x, y, z=-43,-46,24)$ and the left insula $(x, y, z=-43,-1$, 17). The reverse comparison showed tiny clusters located in most posterior temporal regions (see Fig. $6 \mathrm{C} ; x, y, z= \pm 39,-80,23$ ).
The (Group $\times$ Condition) compound contrast ([SWsp-SWac ] cont vs. [SWsp-SWac $]_{\text {dys }}$ ), exploring the effects of Group $\times$ Condition interaction (Fig. 7A) revealed the surplus of activity in the control group relative to the dyslexic group for the SWsp-SWac contrast The brain areas that were more sensitive to phonological perception mode effects in the normal readers compared to dyslexic subjects predominated in the left hemisphere with clusters scattered in the frontal, temporal and parietal lobes (see corresponding clusters

Table 5

Higher activation in the control subjects than in the dyslexic subjects (top) and reverse (bottom) for the sound minus rest comparison

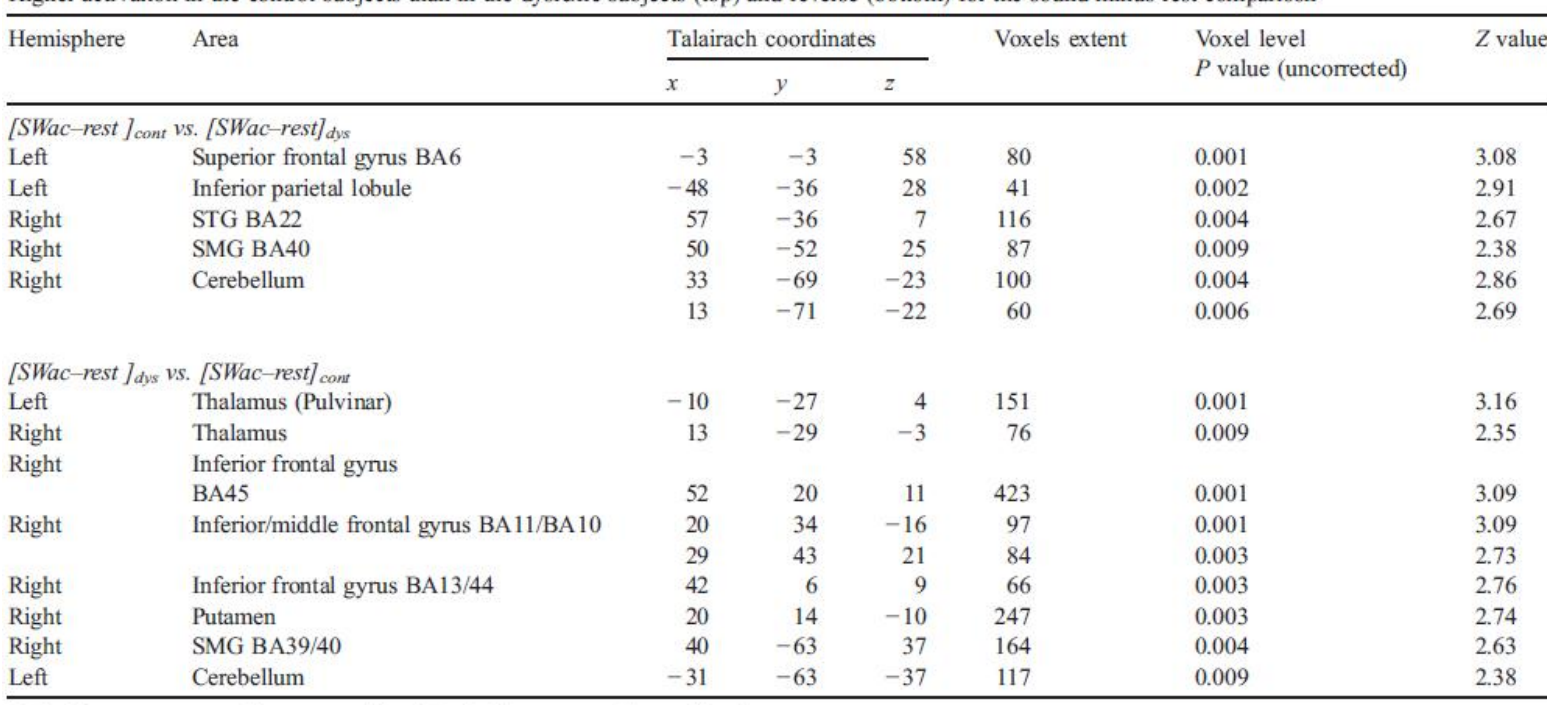

Only clusters corresponding to voxel level $P<0.01$ uncorrected were listed.

Abbreviations as in Table 4. 
A

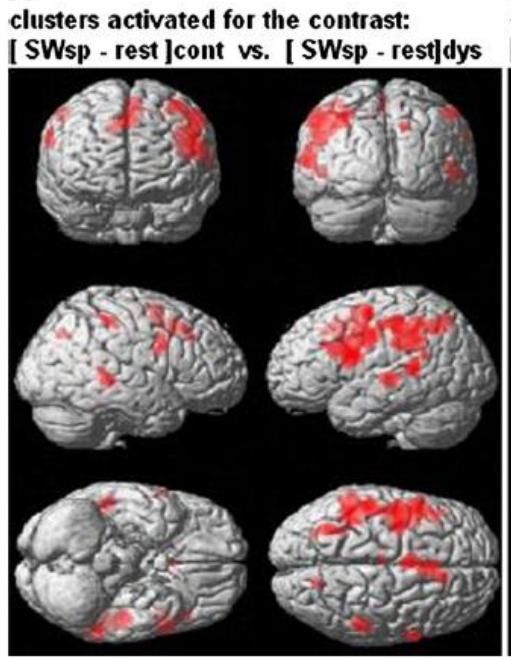

B

clusters activated for the contrast:

[ SWsp - rest ]dys vs. [ SWsp - rest]cont

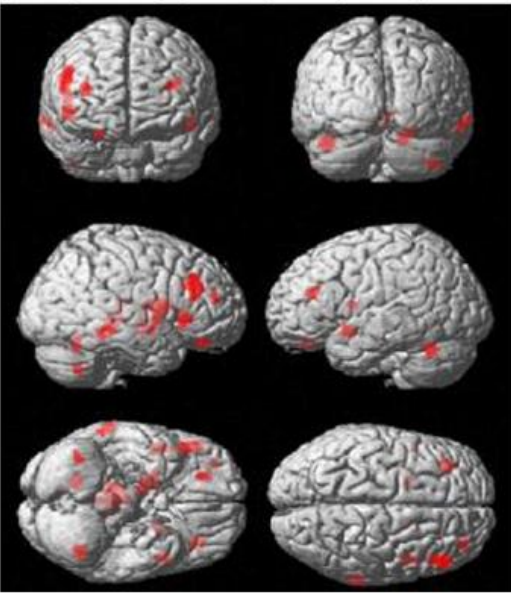

Fig. 5. Main clusters activated in the contrast image SWsp minus Rest. Controls $>$ Dyslexics $(P=0.05)$ inclusively masked by SWsp minus Rest in dyslexics (left) and Dyslexics $>$ Controls $(P=0.05)$ inclusively masked by SWsp minus Rest in controls (right) (abbreviations as in Fig. 2).

located in the left-sided BAs 6, 13/47, 37 and 40 in Table 7). In the right hemisphere, fewer activation clusters were found that were concentrated around the sylvian sulcus and in subcortical structures. Moreover, an activation cluster was also seen in the cingulate cortex.
We hypothesized differential effects of our experimental paradigm in control versus dyslexic subjects in the left parietal region. To further analyze this effect a three-factor (hemisphere, group, perception mode), ANOVA was conducted on CBF values

Table 6

Higher activation in the control subjects than in the dyslexic subjects (top) and reverse (bottom) for the speech minus rest comparison

\begin{tabular}{|c|c|c|c|c|c|c|c|}
\hline \multirow[t]{2}{*}{ Hemisphere } & \multirow[t]{2}{*}{ Area } & \multicolumn{3}{|c|}{ Talairach coordinates } & \multirow[t]{2}{*}{ Voxels extent } & \multirow{2}{*}{$\begin{array}{l}\text { Voxel level } \\
P \text { value (uncorrected) }\end{array}$} & \multirow[t]{2}{*}{$Z$ value } \\
\hline & & $x$ & $y$ & $z$ & & & \\
\hline \multicolumn{8}{|c|}{$[S W s p-r e s t]_{\text {cont }} v s .[S W s p-r e s t]_{d y s}$} \\
\hline \multirow[t]{2}{*}{ Left } & \multirow[t]{2}{*}{ Inferior frontal gyrus/insula/inferior parietal lobule } & -43 & 1 & 17 & 1961 & 0.000 & 4.09 \\
\hline & & -45 & -42 & -32 & & & \\
\hline Left & STG BA22 & -43 & -25 & -2 & 167 & 0.001 & 3.26 \\
\hline Left & MTG BA21 & -50 & -46 & 8 & 155 & 0.002 & 2.92 \\
\hline Right & MTG BA20 & 48 & -38 & -6 & 99 & 0.009 & 2.37 \\
\hline Left & Superior parietal lobule precuneus BA 19 & -29 & -62 & 39 & 173 & 0.002 & 2.93 \\
\hline Right & Inferior parietal lobule BA40 & 45 & -42 & 47 & 49 & 0.005 & 2.56 \\
\hline \multirow[t]{2}{*}{ Left } & Cingulate gyrus/middle frontal gyrus & -6 & -25 & 44 & 33 & 0.007 & 2.47 \\
\hline & $\mathrm{BA} 31 / 6$ & -4 & -1 & 51 & 443 & 0.007 & 2.44 \\
\hline Right & Inferior frontal gyrus BA9/44 & 54 & 4 & 28 & 50 & 0.01 & 2.32 \\
\hline \multicolumn{8}{|c|}{$[S W s p-r e s t]_{d y s} v s .[S W s p-r e s t]_{c o n t}$} \\
\hline \multirow[t]{2}{*}{ Right } & \multirow[t]{2}{*}{ Middle frontal gyrus BA9 } & 43 & 28 & 31 & 166 & 0.000 & 3.30 \\
\hline & & 29 & 43 & 18 & 48 & 0.008 & 2.41 \\
\hline Right & Parahippocampal gyrus & 12 & -36 & -7 & 226 & 0.001 & 3.15 \\
\hline Right & Thalamus & 13 & -5 & 8 & 72 & 0.001 & 3.08 \\
\hline Right & Inferior frontal gyrus BA47 & 43 & 20 & 1 & 95 & 0.002 & 2.85 \\
\hline Left & Putamen & -18 & -1 & 8 & 35 & 0.006 & 2.51 \\
\hline Left & \multirow[t]{2}{*}{ Cerebellum } & -38 & -60 & -28 & 92 & 0.007 & 2.47 \\
\hline Right & & 15 & -63 & -26 & 78 & 0.01 & 2.32 \\
\hline Right & Middle temporal gyrus BA20 & 57 & -38 & -11 & 60 & 0.007 & 2.46 \\
\hline Left & STG & -43 & 4 & -12 & 40 & 0.007 & 2.44 \\
\hline Right & IFG BA11 & 20 & 34 & -20 & 34 & 0.008 & 2.41 \\
\hline Right & Precentral gyrus BA13/BA44 & 43 & 4 & 11 & 107 & 0.008 & 2.40 \\
\hline
\end{tabular}

Only clusters corresponding to voxel level $P<0.01$ uncorrected were listed. Abbreviations as in Table 4 . 
A Condition main effect whatever group (Controls and Dyslexics) $(P=0.001, k=30)$

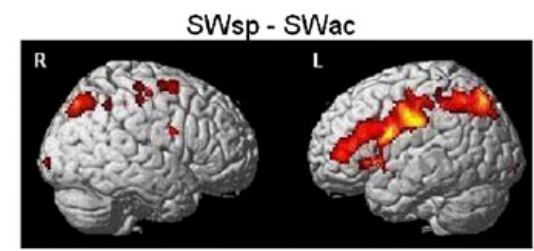

The largest clusters are left sided: 1713 voxels for the parietal one and 2780 voxels for the frontal one.
$Z=5.72$ and $Z=5.45 ; P_{\text {FWE }}=0.001$ and
$P_{\text {FWE }}=0.003$

B Group main effect whatever condition (SWac and SWsp) $(P=0.001, k=30)$

Controls - Dyslexics

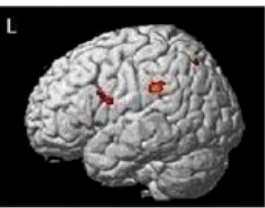

Dyslexics - Controls

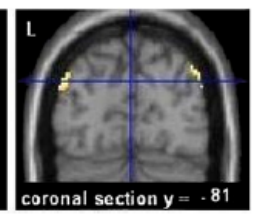

Fig. 6. (A) Condition main effect whatever group, SWsp $>$ SWac. (B) Group main effect whatever condition with Controls $>$ Dyslexics contrast (left) and Dyslexics $>$ Controls (right) $(P=0.001, k=30)$.

recorded at the peak of the left-sided parietal cluster $(x, y, z=-36$ $-4434)$ and the homologous right-sided cluster ( $x, y, z=40-52$ 38). These analyses were graphically depicted in Fig. 7A, bottom-left. A significant main effect of perception mode was found $[F(1,28)=11.89, P=0.001]$ as well as a slight hemisphere main effect $[F(1,28)=4.15, P=0.05]$. A strong interaction between group and perception mode was observed $[F(1,28)=17.01$, $P=0.0003]$. This interaction was due to strong signal increase from SWac to SWsp in the control group bilaterally (paired $t$-test, $P=0.004$, left peak; $P=0.001$, right peak). Post hoc analyses further demonstrated (i) diminished activation in the left-sided region in the speech mode in the dyslexic group relative to the control group (post hoc unpaired $t$-test $P=0.008$ ), and (ii) higher activity in the right parietal region in the dyslexic group relative to the homologous leftsided region whatever conditions (post hoc paired $t$-test $P=0.0001$ ).

The reverse contrast ([SWsp-SWac $]_{\text {dys }}$ vs. [SWsp-SWac $]_{\text {cont }}$ ) (Fig. 7B) showed that signal change between SWsp and SWac was larger in dyslexic subjects than in control subjects in areas located in the medial occipital and inferior temporal cortex, predominantly in the right hemisphere (Fig. 5). However, deactivation was observed in the very same region for the SWac-rest contrast in the dyslexic group (see above Results Section 1.2). To further investigate this phenomenon, an analysis of signal changes relative to rest in SWac and SWsp, respectively, was conducted, in each individual, in the main peak of a cluster located in the right medial occipital cortex BA19 $(x, y, z=10,-96,-12)$. These results were used to compute a (group, condition) ANOVA that showed an effect of condition $[F(1.28)=11.14, P=0.002]$ and a Group $\times$ Condition interaction $[F(1,28)=6.03, P=0.02]$ (see histogram in Fig. 7B). These findings confirmed that the effect observed in the compound contrast came from lowered activity in the SWac condition in dyslexic subjects relative to both rest and SWsp, whereas a slight, non-significant trend to deactivation was found in the control group for both SWac and SWsp relative to rest. Additional small clusters were located in left precentral and right cerebellum regions.

Discussion

Behavioural results

This study showed that a discrimination task for synthetic speech pairs elicited similar pattern of performance in both the dyslexic group and the normal reader group. In the naive stage of the experiment, or its acoustic mode, subjects tended to react to any pair types by giving 'same' responses. In both groups categorical discrimination responses appeared only after debriefing and training. The setting up of categorical perception after debriefing was witnessed by the dramatic increase of the number of 'different' responses to BETWEEN pairs in the speech mode of the experiment ( $\mathrm{SWsp}$ and $\mathrm{SWsp}+\mathrm{F} 0$ ). It is worth of note that, in spite of a slight trend for decrease of the averaged rate of 'same' responses to SAME pairs as the experiment went on, this effect was not significant although more marked in the dyslexic group than in the control group. Whereas the rate of 'different' responses to WITHIN pairs increased significantly from the acoustic to the speech mode of the experiment, it remained well below the $50 \%$ threshold although subjects were encouraged to find out possible differences between heard items. Crucially, an interaction was found between conditions (SWac vs. SWsp) and pair types (BETWEEN vs. WITHIN) so that the increase of the rate of 'different' responses from SWac to SWsp was higher for BETWEEN pairs. Overall, these performance patterns advocate for a general trend to categorical perception strategy in the speech mode in both control and dyslexic subjects. Analysis of reaction times concurs with this interpretation. In both groups, RTs were longer for BETWEEN pairs in the SWac condition and whatever pair types in speech mode conditions. Access to phonological representations, as stable entities stored in perceptual long-term memory, is a prerequisite to categorical perception of speech. The observed increase of processing time might relate to access to phonological representations with which incoming stimuli had to be matched before subject could make a decision; this top-down processing has an additional time cost effect (Kosslyn et al., 1995; Pernet et al., 2004).

In spite of the similarities of performance pattems between the two groups, accuracy scores were higher in the control group than in the dyslexic group. Although moderate, this group effect did not interact with any other factor; this suggests that a basic, qualitative difference exists between the two groups as far as the proficiency of categorical perception is concerned.

In sum, categorical perception strategy had developed over our experiment in both groups although this strategy was more efficient in normal readers than in the adult dyslexics we studied. In these subjects, persistent deficits in many language and memory tasks were readily observable. However, although reduced, their ability to perform a new phonological categorization suggests that incomplete compensation might have alleviated a basic speech perception deficit (Shaywitz and Shaywitz, 2003a,b; Shaywitz et al., 2003). These compensatory mechanisms observed in our adult dyslexic subjects might account for the discrepancy between the present behavioral results and those described with the same behavioral paradigm by Semiclaes et al. (2001). In the latter, a more deviant pattem of performance was found in younger dyslexic subjects since they showed higher rate of 'different' responses to WITHIN pairs in 
A contrast: [SWsp - SWac]cont vs. [SWsp - SWac]dys
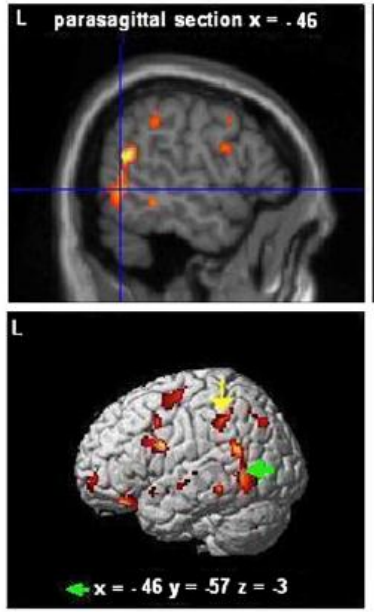

Mean signal recorded in the left parietal
region BA40 [-36-44 34]

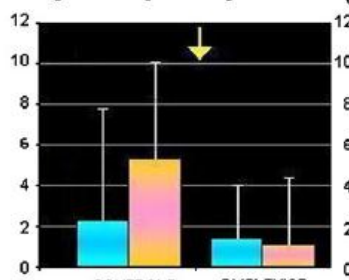

CONTROLS
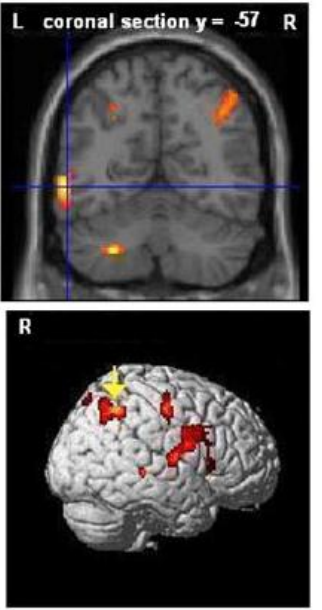

Mean signal recorded in (Superior parietal lobule) [40-52 38]

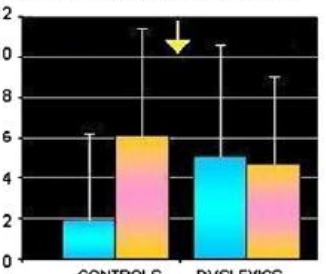

B
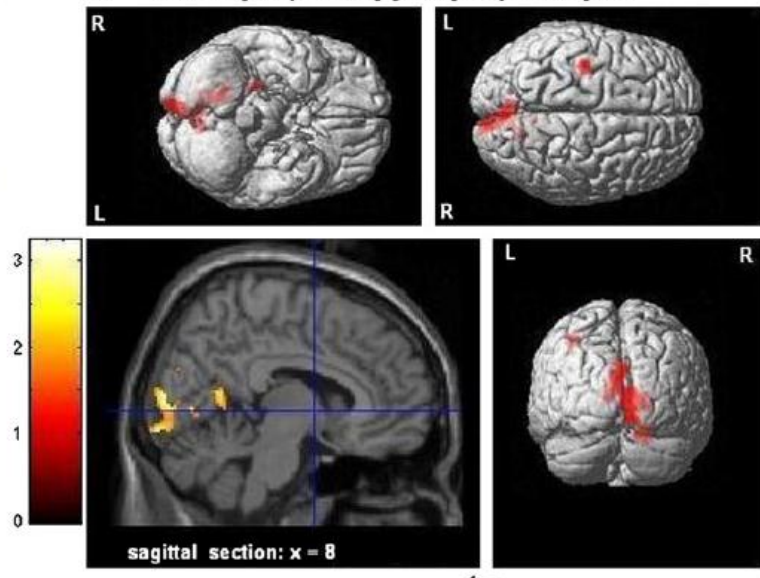

Mean signal recorded in the right medial occipital region $[8,-96,-16]$

for the contrast :

[ISW mask incl(SWsp>Swac)cont.

$\square$ Nonspeech > Rest

$\square$ Speech $>$ Rest

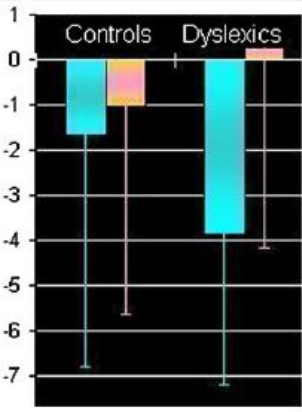

Fig. 7. (A, Top) Main clusters activated in the contrast image SWsp minus SWac. Controls $>$ Dyslexics $(P=0.05)$ inclusively masked with (SWsp minus SWac) contrast in control subjects. (Bottom) Bar charts representing normalized signal mean values in parietal cortex bilaterally $(x, y, z=-36-4434$ and $x, y, z=40$ -52 38) (yellow arrows on rendering above). (B, Top) Main clusters activated in the contrast image SWsp minus SWac, Dyslexics $>$ Controls $(P=0.05)$ inclusively masked with (SWsp minus SWac) contrast in control subjects (Bottom). Bar charts representing normalized signal mean values in the right medial occipital cortex $(x, y, z=8-96-16)$ (abbreviations as in Fig. 2).

the acoustic mode relative to age-matched normal readers, a trend that was not observed in the present study.

\section{PET results}

In the group of normal readers, the PET results showed a leftward asymmetry in the speech mode of the discrimination task while a predominantly right temporal response was elicited by the acoustic mode. By contrast, in the dyslexic group, top-down effects did not result in such a right-to-left shift in the perisylvian areas; the less efficient categorical perception observed in this group involved was associated with diminished activations in the perisylvian regions that was found activated in the control group. These results will be discussed considering in turn effects observed in each group and between groups. For between-group comparisons, results should be considered with some caution as the statistics were not corrected for multiple comparisons; however, the whole study was obtained from a second-level statistical analysis and the reported peaks were well below the considered thresholds.

\section{Normal readers}

We will first address the results observed in the group of normal readers considering the available literature on the brain correlates of phonological perception. The pattern of activation observed in the group of normal readers in the acoustic mode relative to the rest condition involved mainly the posterior part of the right superior temporal gyrus, together with a part of the inferior parietal lobule and the inferior frontal gyrus. This result is consistent with activation observed for the non-phonemic condition of a recent study of categorical phonemic perception in normal subjects (Liebenthal et al., 2005) although these authors reported symmetrical activity in an experiment where subjects were given feedback on the nature of heard stimuli. The rightward preponderance we obtained in the naive subjects could relate to the processing of spectral features of stimuli as non-verbal auditory objects (Zatorre and Belin, 2001).

In the speech mode compared to rest, a massive left-sided activation was observed in three main clusters distributed in the posterior superior temporal, the inferior parietal and the motor and premotor cortex; in the right hemisphere homologous but weaker activations were seen in the inferior parietal and superior temporal regions. The specificity of these effects vis-à-vis phonological topdown processes was assessed by the SWsp-SWac contrast; the perisylvian leftward predominance of activity was identified although it was not as pronounced as might be expected from the lower level comparisons; it concerned mainly the motor/ 
Table 7

Higher activation in the control subjects than in the dyslexic subjects (top) and reverse (bottom) for the speech minus sound comparison

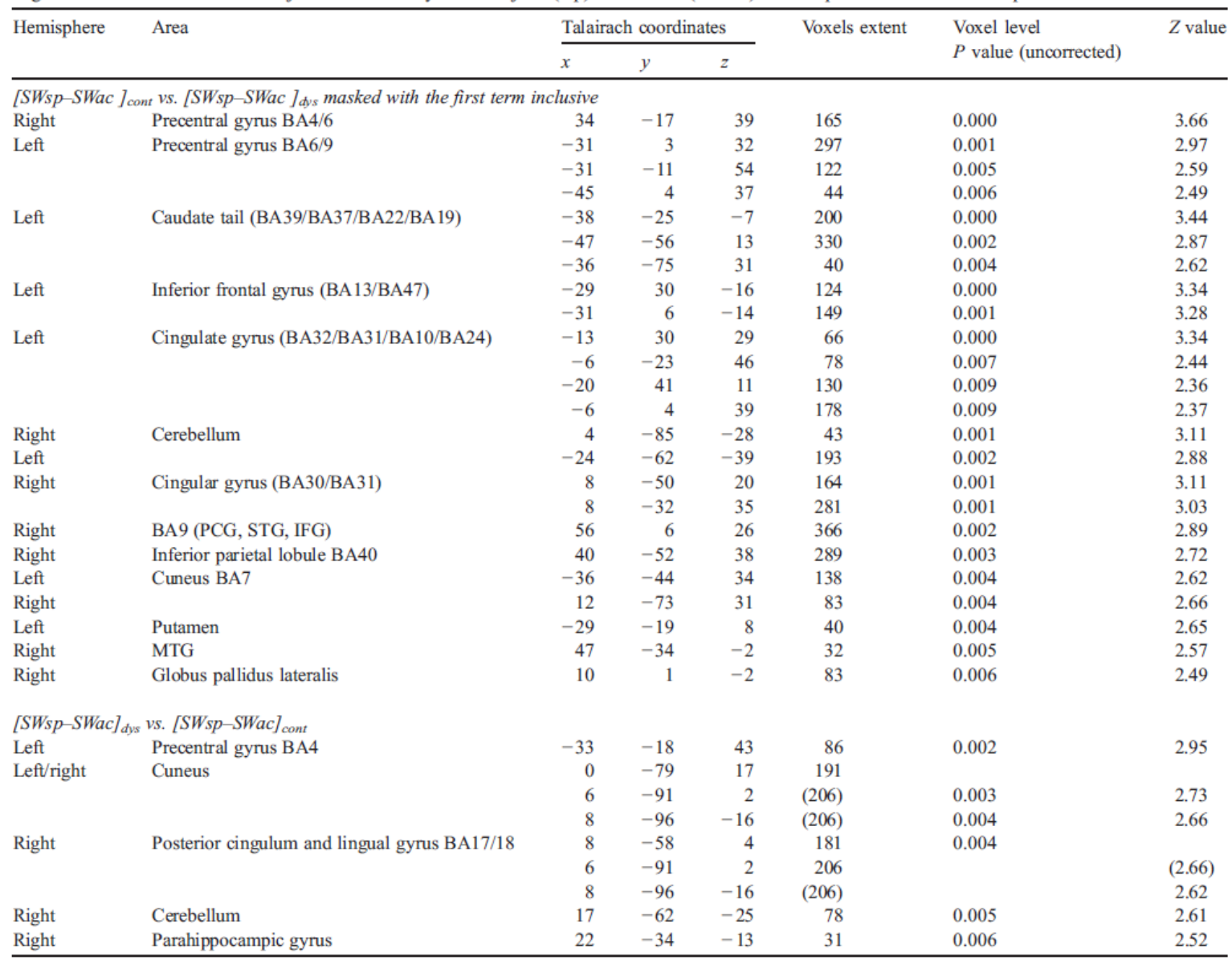

Only clusters corresponding to voxel level $P<0.01$ uncorrected were listed. Abbreviations as in Table 4

premotor cortex and the inferior parietal lobule whereas only a small cluster was observed in the posterior part of the left superior temporal gyrus. Overall, this pattern resembled that described by Dehaene-Lambertz et al. (2005) as far as the comparison between the speech mode against the acoustic mode is concerned (cf. Fig. 5 in the paper of these authors). These convergent findings demonstrate that the brain counterparts of top-down effects for phonemic categorization consist of activation in a set of temporal/ parietal/frontal regions predominating in the left hemisphere.

Concerning the posterior part of the superior temporal gyrus, these findings are in line with the conceptualization of the role of this 'dorsal portion' of the auditory cortex as an interface between auditory and motor representations of speech sounds (Hickok and Poeppel, 2000); however, this region has been found sensitive to both speech and non-speech complex sounds (Celsis et al., 1999; Binder et al., 2005) in both hemispheres. Nevertheless, it may be that increased response in a subpart of this region in the left hemisphere would be elicited by a discrimination task such as that used in Dehaene-Lambertz et al.'s (2005) study and in ours. It has been proposed previously (Wise et al., 2001) that this small part of the posterior superior temporal cortex might have a role as a temporary buffer for phonological contents during speech processing before these information could be transferred to further components of the 'dorsal speech pathway' (for a review, see Demonet et al., 2005).

Activation in the left inferior parietal lobule (mainly in the supra-marginal gyrus) and motor/premotor cortex (BAs 4/6) is likely to reflect respectively the coding of segmented auditory speech streams into abstract phonological representations and their transformation to articulatory counterparts (Hickok and Poeppel, 2000). The activation of the left inferior premotor has been repeatedly described for phonemic processing as for instance a recent study by Katzir et al. (2005).

\section{Dyslexic subjects}

Considering results obtained in the group of dyslexic subjects, the activation observed for the acoustic mode was roughly similar to the pattern found in the control group; however, the rightward predominance was even larger and the involvement of the right inferior frontal gyrus was especially marked as also revealed by the 
between group analysis (cf. infra and Figs. 2 and 4B; Table 5). Another difference concems the deactivation observed in the occipital cortex this effect was marked in the dyslexic group and not significant in the control group (see below). In the speech mode, contrary to what was found in the control group, the rightward preponderance of activation observed in the acoustic mode persisted in the SWsp versus rest contrast. The effects of phonological top-down processes (SWsp vs. SWac contrast) consisted of few left-sided clusters. These clusters, located in the left BA6 and the intra-parietal sulcus, were part of the pattern observed in control readers for the same contrast. SWsp in dyslexic subjects seems therefore to elicit activation in a restricted part of a left-sided functional network relating to phonological processing. This finding is congruent with results of a study by Rumsey et al (1992) or Eden et al. (2004) showing reduced activity in these regions in adult dyslexics compared to control readers during a phonological task.

Increases of activity in normal readers relative to dyslexic subjects. The compound contrast exploring activation in control readers relative to dyslexic subjects for the SWsp-Rest comparison reflected significant activation in both groups and a surplus of activity in the control group. The main clusters were located in the left hemisphere and the dorsal part of the speech-related network, a finding that demonstrates that compared to rest, the speech mode activated this network in both groups though to a larger extent in controls readers.

The specific contribution of the phonological top-down processes to this effect was addressed studying the interaction between mode (SWsp-SWac) and group (control vs. dyslexic) as binary factors. The compound contrast ([SWsp-SWac] $]_{\text {cont }}$ vs. $[\mathrm{SWsp}-\mathrm{SWac}]_{\mathrm{dys}}$ ) inclusively masked by (SWsp-SWac) cont aimed at bringing out areas associated with both significant activation specifically linked to phonological top-down processes in contro subjects and higher activation in the control group than in the dyslexic group. Based on the literature (e.g., Eden et al., 2004), weakening of activation in the dyslexic group was expected in the speech mode relative to the control group. Several clusters were identified in this way.

Some pertain to the above described pattern as the 'dorsal pathway' of the speech-related network. These results show that some subcomponents of these 'dorsal' regions are more sensitive to top-down processes in control readers than in dyslexic subjects. This lack of activation in dyslexic subjects is especially marked in the left parietal cortex. This region was not activated for the SWspSWac contrast when studied in the group of dyslexic subjects only (Fig. 3) and appeared activated in the compound contrast exploring the surplus of activity in the control group relative to the dyslexic group. At variance with control readers, dyslexic subjects failed to show specific engagement of this region during phoneme categorization after debriefing and learning. Defect of activation in the left supra-marginal gyrus has been repeatedly associated with phonological deficit in dyslexia (e.g., Ruff et al., 2002) and the present study shows that the phonological learning paradigm we used brought up neuro-functional correlates that are quite similar to those obtained with more classical tasks such as rhyming tasks (for a review, see Temple, 2002). It is worth noting that studying the group 'main effect' of this interaction allowed us to identify another cluster in the left inferior parietal lobule $(x, y, z=$ $-43,-46,24)$ located just below the cluster involved in the interaction $(x, y, z=-36-4434)$ (Fig. 7A; Table 7). This ventrally located subregion was found more active in the control group than in the dyslexic group in both SWac and SWsp tasks. This finding stresses again the consistency of the functional deficit of this lower part of the left parietal cortex in dyslexia.

While our hypothesis concerned differential effects in the left parietal cortex, this study revealed similar effects in the homologous right-sided parietal cortex with signal increase from SWac to SWsp in control readers (Fig. 7A, bottom). This finding suggests that either top-down phonological processes or training of difficult phonological contrasts in normal subjects might result in the bilateral recruitment of parietal cortex as also suggested by Callan et al. (2003) and by Dehaene-Lambertz et al. (2005) in the previous experiment conducted in normal readers with the stimuli we used in dyslexic subjects the pattern of activation in these regions is drastically different from that seen in normal subjects. While these portions of the parietal cortex were insensitive to change in perception mode, the level of activity was significantly higher on the right hemisphere compared to the left one as also suggested by Simos et al. (2002).

Higher activation in the control group than in the dyslexic group for speech against acoustic mode concerned other clusters, distributed across the posterior part of the left temporal cortex, from BA22 to BA37 (Fig. 7A). The left BA37 has already been shown to activate less in dyslexic subjects than in control subjects in a reading aloud task (Paulesu et al., 2001); a voxel-based morphometry study has recently revealed that this region was characterized, in the same subject samples, by abnormal grey matter density in dyslexic subjects (Silani et al., 2005). Moreover, a categorization task brought out selective activation of this region for Latin letters compared to geometrical shapes and unknown letters (Pemet et al., 2004, 2005). Moreover, McCrory et al. (2005) showed that in dyslexic subjects a functional deficit exists in the left BA37 not only for reading tasks but also for naming visually presented object drawings. The present study considered together with these previous results therefore suggests that modalityindependent effects might exist in this region for language tasks so that dyslexic subjects would show diminished activation relative to that observed in control readers whatever the tasks, at least those involving sublexical processing (e.g., phoneme/grapheme association). This possible a-modal deficit might be a consequence of developmental abnormalities of the cortical structure in this region. It should be noted however that the present study, reported on results of an auditory speech task, and previously mentioned studies dealt with reading tasks, and congruent effects only concerned a restricted zone of partial overlap. Studies involving reading tasks mentioned above did not reveal any group difference in the superior temporal gyrus (BA22) while in turn, the present study did not show differential effects in the lower part of BA37, a key region for reading tasks.

Finally overactivations in the control group relative to the dyslexic group were also observed in the inferior frontal cortex bilaterally. The involvement of the left inferior frontal region in this pattern is in accordance with the well-acknowledged role of left premotor areas in phonological processing (Bookheimer, 2002). Moreover, another important overactivated cluster was located in the right BA6. (A) Many neuroimaging studies have described activation in the right premotor cortex and linked this finding to working memory function and attentional resources that subjects engaged to solve cognitive tasks. Our task consisted in judgment of pairs of heard syllables; we previously showed that when working memory load increased for pairs of heard verbal items, enlarged 
and delayed neural activities were observed in these regions bilaterally (Thierry et al., 2003). A number of neuroimaging studies have investigated the neural substrates of working memory, whatever verbal or visuospatial (e.g., Smith and Jonides, 1999). In more specific terms, one might propose that in the speech mode normal readers were able to accurately categorize stimuli as (i) they learnt how to map stimuli to the corresponding phonological prototypes stored in perceptual long-term memory, and (ii) they processed identified stimuli via verbal working memory. Krishnan et al. (2005), in their study, described the involvement of this frontal region when comparing phoneme processing in mother tongue (English) and in a new language (Chinese). Callan et al. (2003, 2004) emphasized the role of the perceptual/motor loop for learning new phonological categories, a function that was associated with strong activation in the right motor/premotor frontal cortex. Several other studies concur with this interpretation (Chee et al., 2001; Dehaene et al., 1997; Kim et al., 1997; Nakai et al., 1999).

Increases of activity in dyslexic subjects relative to normal readers. The most striking result was an apparent surplus of activity in the dyslexic group relative to the control readers for the SWsp-SWac comparison, seen in the right medial occipital cortex, i.e., the primary visual cortex and the early association areas. This effect was also observed for the same contrast within the dyslexic group. However, this activation was not observed when SWsp was compared to rest. Further analyses revealed that this effect related mainly to a strong deactivation in these regions for SWac in dyslexic subjects who also showed a very mild trend to activation in this region for SWsp.

Deactivation of the visual cortex during an auditory task has been described already and interpreted as a cross-modal interaction between auditory and visual cortex, in normal subjects (Laurienti et al., 2002). We show that this deactivation is much more marked in dyslexic subjects but the neural underpinnings of this phenomenon remain to be understood. It might relate to an intense focalization of attention resources in the auditory domain during the (demanding) SWac task resulting in a cross-modal deactivation in the visual cortex. A trend to increase of activity in the visual cortex during the speech mode might suggest that the inhibitory effect due to auditory input could be counter-balanced by a topdown effect following phoneme categorization learning. Possible mechanisms of this language-induced top-down modulation might be linked to mental imagery, as some dyslexic subjects might be prone to evoke visual representations of heard syllables. In neuroimaging studies of deaf patients who benefited from cochlear implants, speech stimulation was shown to elicit activation in the early visual areas in the right hemisphere whereas noises did not have such an effect (Giraud et al., 2001; Giraud and Truy, 2002) The authors hypothesized that these findings reflect the involvement of mental imagery, as subjects likely resorted to the visualization of either the corresponding printed words or the corresponding speaking faces, the latter relating to special proficiency of lip reading in deaf subjects. Moreover, activation of the medial visual cortex during speech processing has already been described in a brief report of a single-case study of a dyslexic patient presenting with a particularly severe phonological deficit (Habib et al., 1996). One might speculate that a trend to activation in the visual areas while dyslexic subjects processed SWsp sounds reflects an accessory strategy according to which visual representations would help deciphering laboriously perceived ambiguous speech sounds. This hypothesis remains to be further confirmed by dedicated experiments.

Finally in the dyslexic group, several clusters were found in the right middle frontal gyrus and the right inferior frontal gyrus in both its lateral and orbital parts. The latter was more clearly activated in the compound contrasts opposing the dyslexic group to the control group, for both comparisons opposing each task to rest (Figs. 4B and 5B; Tables 5 and 6). The right premotor areas has been found activated in several neuroimaging experiments on speech perception and has been linked to increased demand on focused attention to speech (Binder, 2000; Binder et al., 2000); and activation in this region has also been reported in infants exposed to connected speech only in awakened phases (Dehaene-Lambertz et al., 2002). The orbital part of the inferior frontal gyrus has been related to many aspects of task solving experiments, especially to the effects of guessing and/or anxiety in subjects facing difficult tasks (e.g., Elliott et al., 2000). It may be that, even in the acoustic mode, dyslexic subjects experienced difficulties performing the discrimination task and engaged attention-related areas, especially the right premotor cortex.

In conclusion, close-to-normal performance on learning phoneme categorization in dyslexic subjects imply that some sort of compensatory mechanisms had taken place in these subjects although specific neuropsychological assessment readily demonstrated the persistence of a typical pattern of language and memory deficits. Studies of the neural bases of compensatory mechanisms in young adult dyslexics have already been performed (Simos et al., 2002; Shaywitz et al., 2003), suggesting respectively changes in hemispheric preponderance for language functions and changes in connectivity patterns across key regions. The present study based on planned contrasts between conditions does not provide clear evidence of compensatory mechanisms implicating for instance areas in the right hemisphere. We think that an important way to explore compensatory processes is to perform correlational analyses between performance changes and hemodynamic changes that will be described elsewhere. However, the overactivation in the right inferior frontal cortex in the dyslexic group seems to relate mainly to engagement of effortful processing during the discrimination task even in the acoustic mode. Lastly, this study confirms previous findings on hypofunction of several regions in the left hemispheric cortex, the supra-marginal gyrus and the junction of temporal/occipital cortex, in dyslexia.

\section{Acknowledgments}

This study was supported by the French 'Programme Interdisciplinaire, Cognition et Traitement de l'Information (CNRS), CT101-53' headed by Liliane Sprenger-Charolles and Willy Serniclaes; a special thanks to the technical staff of the Toulouse PET centre and to a friend, Marie-Pierre Dupont.

\section{References}

Binder, J., 2000. The new neuroanatomy of speech perception. Brain 123 (Pt. 12), 2371-2372.

Binder, J.R., Frost, J.A., Hammeke, T.A., Bellgowan, P.S., Rao, S.M., Cox, R.W., 1999. Conceptual processing during the conscious resting state. A functional MRI study. J. Cogn. Neurosci. 11, 80-95.

Binder, J.R., Frost, J.A., Hammeke, T.A., Bellgowan, P.S., Springer, J.A., Kaufman, J.N., Possing, E.T., 2000. Human temporal lobe activation by speech and nonspeech sounds. Cereb. Cortex 10, 512-528. 
Binder, J.R., Medler, D.A., Desai, R., Conant, L.L., Liebenthal, E., 2005. Some neurophysiological constraints on models of word naming. NeuroImage 27, 677-693.

Bookheimer, S., 2002. Functional MRI of language: new approaches to understanding the cortical organization of semantic processing. Annu. Rev. Neurosci. 25, 151-188.

Callan, D.E., Tajima, K., Callan, A.M., Kubo, R., Masaki, S., AkahaneYamada, R., 2003. Learning-induced neural plasticity associated with improved identification performance after training of a difficult secondlanguage phonetic contrast. NeuroImage 19, 113-124.

Callan, D.E., Jones, J.A., Callan, A.M., Akahane-Yamada, R., 2004. Phonetic perceptual identification by native- and second-language speakers differentially activates brain regions involved with acoustic phonetic processing and those involved with articulatory-auditory/ orosensory internal models. Neurolmage 22, 1182-1194.

Celsis, P., Boulanouar, K., Doyon, B., Ranjeva, J.P., Berry, I., Nespoulous, J.L., Chollet, F., 1999. Differential fMRI responses in the left posterior superior temporal gyrus and left supramarginal gyrus to habituation and change detection in syllables and tones. Neurolmage 9, 135-144.

Chee, M.W., Hon, N., Lee, H.L., Soon, C.S., 2001. Relative language proficiency modulates BOLD signal change when bilinguals perform semantic judgments. Blood oxygen level dependent. Neurolmage 13 , $1155-1163$.

Dehaene, S., Dupoux, E., Mehler, J., Cohen, L., Paulesu, E., Perani, D., van de Moortele, P.F., Lehericy, S., Le Bihan, D., 1997. Anatomical variability in the cortical representation of first and second language. NeuroReport 8, 3809-3815.

Dehaene-Lambertz, G., Dehaene, S., Hertz-Pannier, L., 2002. Functional neuroimaging of speech perception in infants. Science 298, 2013-2015.

Dehaene-Lambertz, G., Pallier, C., Semiclaes, W., Sprenger-Charolles, L., Jobert, A., Dehaene, S., 2005. Neural correlates of switching from auditory to speech perception. Neurolmage 24, 21-33.

Demonet, J.F., Taylor, M.J., Chaix, Y., 2004. Developmental dyslexia Lancet 363, 1451-1460.

Demonet, J.F., Thierry, G., Cardebat, D., 2005. Renewal of the neurophysiology of language: functional neuroimaging. Physiol. Rev. $85,49-95$.

Eden, G.F., Jones, K.M., Cappell, K., Gareau, L., Wood, F.B., Zeffiro, T.A., Dietz, N.A., Agnew, J.A., Flowers, D.L., 2004. Neural changes following remediation in adult developmental dyslexia. Neuron 44 , $411-422$.

Elliott, R., Dolan, R.J., Frith, C.D., 2000. Dissociable functions in the medial and lateral orbitofrontal cortex: evidence from human neuroimaging studies. Cereb. Cortex 10, 308-317.

Frith, U., 1999. Paradoxes in the definition of dyslexia. Dyslexia 5 , 192-214.

Giraud, A.L., Truy, E., 2002. The contribution of visual areas to speech comprehension: a PET study in cochlear implants patients and normalhearing subjects. Neuropsychologia 40, 1562-1569.

Giraud, A.L., Price, C.J., Graham, J.M., Truy, E., Frackowiak, R.S., 2001. Cross-modal plasticity underpins language recovery after cochlear implantation. Neuron 30, 657-663.

Giraud, K., Demonet, J.F., Habib, M., Marquis, P., Chauvel, P., LiegeoisChauvel, C., 2005. Auditory evoked potential patterns to voiced and voiceless speech sounds in adult developmental dyslexics with persistent deficits. Cereb. Cortex 15, 1524-1534.

Gusnard, D.A., Raichle, M.E., Raichle, M.E., 2001. Searching for a baseline: functional imaging and the resting human brain. Nat. Rev., Neurosci. 2, 685-694.

Habib, M., Demonet, J.F., Frackowiak, R., 1996. Cognitive neuroanatomy of language: contribution of functional cerebral imaging. Rev. Neurol. (Paris) 152, 249-260.

Hickok, G., Poeppel, D., 2000. Towards a functional neuroanatomy of speech perception. Trends Cogn. Sci. 4, 131-138.

Katzir, T., Misra, M., Poldrack, R.A., 2005. Imaging phonology without print: assessing the neural correlates of phonemic awareness using fMRI. NeuroImage 27, 106-115.
Kim, K.H., Relkin, N.R., Lee, K.M., Hirsch, J., 1997. Distinct cortical area associated with native and second languages. Nature 388, 171-174.

Kosslyn, S.M., Alpert, N.M., Thompson, W.L., 1995. Identifying objects at different levels of hierarchy: a positron emission tomography study. Hum. Brain Mapp. 3, 107-132.

Krishnan, A., Xu, Y., Gandour, J., Cariani, P., 2005. Encoding of pitch in the human brainstem is sensitive to language experience. Brain Res. Cogn. Brain Res. 25, 161-168.

Labatut, V., Pastor, J., Ruff, S., Demonet, J.F., Celsis, P., 2004. Cerebral modeling and dynamic Bayesian networks. Artif. Intell. Med. 30, 119-139.

Laurienti, P.J., Burdette, J.H., Wallace, M.T., Yen, Y.F., Field, A.S., Stein, B.E., 2002. Deactivation of sensory-specific cortex by cross-modal stimuli. J. Cogn. Neurosci. 14, 420-429.

Liberman, A.M., Harris, K.S., Hoffman, H.S., Griffith, B.C., 1957. The discrimination of speech sounds within and across phoneme boundaries. J. Exp. Psychol. 54, 358-368.

Liebenthal, E., Binder, J.R., Spitzer, S.M., Possing, E.T., Medler, D.A., 2005. Neural substrates of phonemic perception. Cereb. Cortex 15 , $1621-1631$.

McCrory, E., Frith, U., Brunswick, N., Price, C., 2000. Abnormal functional activation during a simple word repetition task: a PET study of adul dyslexics. J. Cogn. Neurosci. 12, 753-762.

McCrory, E.J., Mechelli, A., Frith, U., Price, C.J., 2005. More than words: a common neural basis for reading and naming deficits in developmental dyslexia? Brain 128, 261-267.

Nakai, T., Matsuo, K., Kato, C., Matsuzawa, M., Okada, T., Glover, G.H., Moriya, T., Inui, T., 1999. A functional magnetic resonance imaging study of listening comprehension of languages in human at 3 teslacomprehension level and activation of the language areas. Neurosci. Lett. 263, 33-36.

Paulesu, E., Demonet, J.F., Fazio, F., McCrory, E., Chanoine, V., Brunswick, N., Cappa, S.F., Cossu, G., Habib, M., Frith, C.D., Frith, U., 2001. Dyslexia: cultural diversity and biological unity. Science 291, 2165-2167.

Pernet, C., Franceries, X., Basan, S., Cassol, E., Demonet, J.F., Celsis, P., 2004. Anatomy and time course of discrimination and categorization processes in vision: an fMRI study. Neurolmage 22, 1563-1577.

Pernet, C., Celsis, P., Demonet, J.F., 2005. Selective response to letter categorization within the left fusiform gyrus. Neurolmage 28, 738-744.

Ruff, S., Cardebat, D., Marie, N., Demonet, J.F., 2002. Enhanced response of the left frontal cortex to slowed down speech in dyslexia: an fMRI study. NeuroReport 13, 1285-1289.

Ruff, S., Marie, N., Celsis, P., Cardebat, D., Demonet, J.F., 2003. Neural substrates of impaired categorical perception of phonemes in adult dyslexics: an fMRI study. Brain Cogn. 53, 331-334.

Rumsey, J.M., Andreason, P., Zametkin, A.J., Aquino, T., King, A.C., Hamburger, S.D., Pikus, A., Rapoport, J.L., Cohen, R.M., 1992. Failure to activate the left temporoparietal cortex in dyslexia. An oxygen 15 positron emission tomographic study. Arch. Neurol. 49, 527-534.

Serniclaes, W., Sprenger-Charolles, L., Carre, R., Demonet, J.F., 2001. Perceptual discrimination of speech sounds in developmental dyslexia. J. Speech Lang. Hear. Res. 44, 384-399.

Serniclaes, W., Van Heghe, S., Mousty, P., Carre, R., Sprenger-Charolles, L., 2004. Allophonic mode of speech perception in dyslexia. J. Exp. Child Psychol. 87, 336-361.

Shaywitz, S.E., Shaywitz, B.A., 2003a. Dyslexia (specific reading disability). Pediatr. Rev. 24, 147-153.

Shaywitz, S.E., Shaywitz, B.A., 2003b. The science of reading and dyslexia J. AAPOS 7, 158-166

Shaywitz, S.E., Shaywitz, B.A., Fulbright, R.K., Skudlarski, P., Mencl, W.E., Constable, R.T., Pugh, K.R., Holahan, J.M., Marchione, K.E., Fletcher, J.M., Lyon, G.R., Gore, J.C., 2003. Neural systems for compensation and persistence: young adult outcome of childhood reading disability. Biol. Psychiatry 54, 25-33.

Silani, G., Frith, U., Demonet, J.F., Fazio, F., Perani, D., Price, C., Frith, C.D., Paulesu, E., 2005. Brain abnormalities underlying altered activation in dyslexia: a voxel based morphometry study. Brain 128, 2453-2461.

Simos, P.G., Breier, J.I., Fletcher, J.M., Bergman, E., Papanicolaou, A.C., 
2000. Cerebral mechanisms involved in word reading in dyslexic children: a magnetic source imaging approach. Cereb. Cortex 10, 809-816.

Simos, P.G., Fletcher, J.M., Bergman, E., Breier, J.I., Foorman, B.R. Castillo, E.M., Davis, R.N., Fitzgerald, M., Papanicolaou, A.C., 2002. Dyslexia-specific brain activation profile becomes normal following successful remedial training. Neurology 58, 1203-1213.

Smith, E.E., Jonides, J., 1999. Storage and executive processes in the frontal lobes. Science 283, 1657-1661.

Sprenger-Charolles, L., Colé, P., Serniclaes, W., 2006. Reading Acquisition and Developmental Dyslexia. Psychology Press.
Temple, E., 2002. Brain mechanisms in normal and dyslexic readers. Curr. Opin. Neurobiol. 12, 178-183.

Thierry, G., Ibarrola, D., Demonet, J.F., Cardebat, D., 2003. Demand on verbal working memory delays haemodynamic response in the inferior prefrontal cortex. Hum. Brain Mapp. 19, 37-46.

Wise, R.J., Scott, S.K., Blank, S.C., Mummery, C.J., Murphy, K., Warburton, E.A., 2001. Separate neural subsystems within 'Wernicke's area'. Brain 124, 83-95.

Zatorre, R.J., Belin, P., 2001. Spectral and temporal processing in human auditory cortex. Cereb. Cortex 11, 946-953. 